\title{
UN CARNET DE LECTURE A PLUSIEURS VOIX
}

\author{
Anne JORRO \\ Université de Provence \\ UMR-ADEF
}

\begin{abstract}
Résumé
Une formation expérientielle à la lecture littéraire a été proposée dans un cours de maîtrise dans le but de favoriser l'articulation entre les apports théoriques et la pratique effective de lecteur interprète. Le roman «Le soleil des mourants » de Jean-Claude Izzo étant choisi, dixhuit lecteurs ont interagi par écrit dans un carnet de lecture sur une période de deux mois. Dans cette situation, l'activité langagière est particulièrement sollicitée de manière à ce que chaque lecteur énonce son positionnement à l'égard du roman, précise ses clés de lecture, argumente de leur bien fondé lorsqu'un débat interprétatif est en jeu. Les traces écrites sont lues, relues, commentées par les lecteurs; progressivement les échanges gagnent en réflexivité et il devient possible de saisir le mouvement interprétatif suscité par la lecture du roman. A l'issue de cette expérience, les lecteurs sont en mesure d'évaluer leur posture de lecteur et d'identifier ce qui relèverait d'une posture de lecteur interprète.
\end{abstract}

Most clés : lecture littéraire, entour didactique, carnet de lecture, dialogisme, posture de lecteur interprète, hétérogénéité interprétative, formation expérientielle.

La formation à la lecture littéraire, en pleine expansion auprès des enseignants du primaire, oriente ses apports didactiques sur le nécessaire passage des pratiques explicatives aux approches interprétatives. L'offre de formation concerne l'explicitation des théories de la lecture littéraire, l'analyse de situations didactiques, en particulier, les travaux relatifs aux cercles de lecture (Terwagne, Vanhulle \& Lafontaine, 2003), l'analyse de séquences littéraires filmées en classe ou encore l'analyse de corpus de lecteurs novices et confirmés. Dans ces différentes stratégies de formation, la dimension analytique est particulièrement valorisée. Or, il nous paraît tout aussi important de penser la formation didactique du point de vue du rapport à la lecture littéraire et de construire des dispositifs de formation ad hoc. Dans la présente recherche, une modalité de formation inspirée des démarches de formation expérientielle (Courtois \& Pineau 1991 ; Bourassa \& alii 1999) a été mise en œuvre de telle sorte que les étudiants (enseignants, maîtres-formateurs, conseillers pédagogiques, futurs PE) entrent dans un processus interprétatif à partir de la lecture d'un roman et laissent des traces écrites de cette lecture dans un carnet sur une période de deux mois. Ainsi, la formation à la lecture littéraire sollicite l'activité langagière des formés, notamment en instaurant une écriture partagée ouverte à la pluralité des interprétations, activité aboutissant à une approche évaluative du processus de formation. 
Le dispositif de formation répondait à une triple exigence :

- D'une part, l'expérience de lecture situait les lecteurs dans le registre de l'action et la formation didactique trouvait un équilibre entre les apports théoriques et l'expérimentation proprement dite,

- D'autre part, le fait d'entrer dans une écriture partagée à propos de processus interprétatifs sensibilisait les lecteurs à l'hétérogénéité interprétative, à la pluralité des formes de réception,

- Enfin, l'activité langagière suscitait la réflexivité des lecteurs. Les échanges entre lecteurs offraient la possibilité d'évoquer une émotion, de la reformuler, mais aussi d'entrer dans un conflit interprétatif en défendant un point de vue. Cette activité langagière permettant alors de se positionner à un niveau méta pour saisir les registres de lecture et ainsi d'identifier la circulation des postures de lecteur.

Dans cet article, je chercherai à mettre en évidence l'impact des pratiques langagières dans le processus interprétatif, en particulier, à analyser le mouvement interprétatif à partir des compétences délibératives des lecteurs, qu'elles s'inscrivent sur fond de consensus ou de conflit de valeurs.

\section{Entour et matérialité dans le dispositif de formation}

Le dispositif de formation est pensé comme un dispositif d'amplification du processus interprétatif. Des actes particulièrement visibles sont recherchés dans le fait de choisir collectivement un ouvrage et d'argumenter sur le bien fondé du choix, mais aussi dans le fait de se lancer dans une lecture collective, d'écrire ses réflexions à propos du roman et d'interagir avec d'autres lecteurs dans un carnet de lecture. La matérialité de l'écriture offerte par le carnet contrebalance la fugacité du processus interprétatif. Ainsi, les interactions langagières ont l'avantage de laisser des traces et les lecteurs ne manquent pas de se lire et de s'interpeller dans le carnet. L'écriture favorise une mise en relief de l'activité interprétative. Dans ce dispositif de formation, l'entour didactique (François, 1998), constitué des phases préliminaires, favoriserait le cheminement progressif des formés dans la démarche interprétative (Jorro, 2001).

\subsection{Le lancement}

Au cours du premier semestre 2003, le cours de didactique du français, en maîtrise, commence et les étudiants sont informés de la dynamique de travail : apports de références théoriques, expérimentation d'une démarche de lecture interprétative en passant par l'écriture et le débat oral une fois que les lecteurs ont fait l'expérience de l'écriture partagée.

Le projet de formation explicité et l'effet de surprise passé, les étudiants reviennent dès la seconde séance avec des ouvrages qu'ils souhaitent proposer au groupe de lecteurs. Un premier tri est fait mais il est convenu d'attendre la séance suivante pour élargir l'offre et permettre un choix qui rencontre l'adhésion du plus grand nombre. A la troisième séance, deux livres suscitent un réel intérêt «Soie» de Alessandro Baricco et «Le soleil des mourants » de Jean-Claude Izzo. Finalement, le groupe d'étudiants de maîtrise choisit le second ouvrage. Les raisons du choix sont nettement subjectives: si ce livre a retenu l'attention des lecteurs c'est en partie parce qu' il s'agissait de découvrir un auteur de la région et que le thème du roman relatant la vie d'un homme en décrochage social sur fond de misères quotidiennes de la rue paraissait «insolite ». Deux étudiants avaient déjà lu le livre, quatre ou cinq lecteurs connaissaient l'auteur et formulaient des appréciations positives. Il 
était plausible que ces orientations subjectives affecteraient les positionnements interprétatifs des lecteurs, que le registre émotif - réactif serait mobilisé et que des conflits de valeurs surgiraient à la lecture de certains passages du livre.

Bien avant l'expérience lecturale proprement dite, la dimension axiologique (Jouve, 2001) traversait les discours. Dès les seuils du texte (Genette, 1987), les lecteurs étaient impliqués dans leur protocole de lecture. En tant que chercheur, nous savions que les interactions langagières seraient inévitablement porteuses de ces positionnements et que le carnet de lecture accueillerait des traces de conflits interprétatifs.

\subsection{Le carnet de lecture}

Le carnet est utilisé avec la consigne suivante : « lieu d'échange autour d'un livre, le carnet de lecture est ouvert à toute pensée, à toute discussion. Interagir au moins quatre fois ».

Le carnet s'échange entre étudiants tandis que la formatrice le voit circuler sans intervenir. La posture de grand lecteur (Jorro, 1999) est totalement absente du dispositif. Il s'agit de favoriser les interactions langagières entre pairs, ce qui renvoie à une démarche de type socio - constructiviste dans laquelle l'expression des points de vue appelle des argumentations, des réfutations, une activité langagière particulièrement féconde dans le déroulement d'une interprétation collective. Le carnet est rendu au bout de deux mois, une photocopie est remise à chaque lecteur et un cercle de lecture est organisé pour la séance suivante. Le carnet de lecture se présente de la manière suivante: la première page du carnet est réservée à l'initiateur d'une réflexion, la page qui suit sert à la discussion. Voici le début du carnet de lecture.

\begin{tabular}{|c|c|}
\hline Réflexion & Discussion \\
\hline $\begin{array}{l}20 \text { Octobre } \\
\text { - En lisant les premières pages du livre j'ai eu } \\
\text { l'impression d'entendre les mots comme prononcés } \\
\text { avec une certaine gouaille. Je ne sais pas si c'est mon } \\
\text { interprétation ou un style voulu par l'auteur. } \\
\text { - je suis frappée par la description faite de la vie dans la } \\
\text { rue. J'en retiens une exacerbation des sentiments liés à } \\
\text { la vie, à la mort. Il y a d'un côté une extraordinaire } \\
\text { liberté à être SDF et en même temps, il y a, encore plus } \\
\text { réelle, l'idée de la mort, de la fin inéluctable, peut-être } \\
\text { accélérée, car ce choix de vie ( conscient ou non) peut } \\
\text { être une forme de lent suicide. }\end{array}$ & $\begin{array}{l}\text { 22/10 réponse à la réflexion } 1 \\
\text { J'ai éprouvé le même sentiment, presque oppressant, } \\
\text { par contre, je pense que cet effet est dû aux choix des } \\
\text { mots et à la structure même du livre. Avant de lire les } \\
\text { premières pages du roman, le lecteur est conditionné } \\
\text { par une note au lecteur frappante, touchante, le lecteur } \\
\text { ne peut que se sentir concerné et petit à petit impliqué } \\
\text { dans le roman. (Christelle) } \\
23 / 10 \text { réponse à la réflexion } 2 \\
\text { Contrairement à la première réflexion, je n'ai pas tenu } \\
\text { compte des mots prononcés. J'ai été complètement } \\
\text { prise par l'ambiance du texte, ce froid. Ce froid qui } \\
\text { installe le décor et qui annonce la mort. Et pourtant ce } \\
\text { froid, que Titi portait en lui et Rico aussi, déborde de } \\
\text { chaleur humaine. Ce texte bascule dans le froid, la } \\
\text { solitude Rico....(Violaine) }\end{array}$ \\
\hline
\end{tabular}

\section{Enjeux didactiques}

La formation à la lecture littéraire commence avec la présentation des conceptions de la lecture : les théories de la réception Jauss (1978), d'Iser (1985), les approches herméneutiques (Ricoeur (1986), les théories de la dissémination du sens (Barthes, 1977, 1973), de la lecture comme jeu (Picard, 1986)... Ces fondements théoriques repris en didactique de la littérature permettent de mettre en évidence des positionnements précis. Ainsi, la lecture littéraire est pensée comme un lieu de tensions (Bucheton, 2002 et Dufays, 2002), de prolifération des significations (Tauveron, 2002). Les postures de lecteur mises en évidence par la recherche 
(Bucheton, 2002, Jorro, 1999, Tauveron, 2002) sont convoquées pour souligner l'activité lectrice et interroger le principe d'un rapport à la lecture, puis celui du rapport à la lecture littéraire.

Ainsi, l'entrée dans la lecture littéraire est réfléchie du point de vue

- du lecteur rétif à l'illusion référentielle, emporté par un souci de vraisemblance, déclinant alors une approche pragmatique dans laquelle le réel l'emporte sur toute perspective fictionnelle,

- du lecteur captif des bons sentiments du roman s'inscrivant dans une posture émotive, empathique parfois militante, débordant largement le monde du roman pour imposer son propre monde,

- du lecteur coopératif entrant dans le roman et manifestant une certaine connaissance du fonctionnement de l'écriture,

- du lecteur lettré orienté vers les questions de style, de forme discursive mais aussi connaisseur des oeuvres littéraires.

- Du lecteur interprète, circulant entre ces postures tout en étant averti des dérives potentielles des deux premières postures.

Si les modèles théoriques de la lecture littéraire importent, il reste encore à préciser le rôle joué par les pratiques langagières (Bautier, 1998) dans la situation de lecture. La démarche socio - constructiviste prend une place essentielle dans le dispositif de formation, en particulier, les interactions langagières sont sollicitées pour accroître la réflexivité des lecteurs. Les jeux de la pensée et du langage (Vygostsky, 1985) sont observables dans le carnet de lecture, les significations se négocient progressivement après de nombreuses reprises, reformulations, déplacements de significations, interpellations conflictuelles. Le dispositif de lecture-écriture partagée fait une large place au dialogisme bakhtinien (1984), encourage les interpellations puisque les lecteurs disposent d'une durée de deux mois pour proposer leur réflexion. Cette dimension socio - constructiviste nous parait particulièrement observable lorsqu'un conflit d'interprétation surgit incitant les lecteurs à avancer des arguments. Le pathos qui court sur certaines pages du carnet est vivement retourné par l'ironie d'un lecteur qui fondera son argumentation sur une série de clés de lecture dont certains pairs s'empareront par la suite pour en renverser la force persuasive. Nous utiliserons la notion de clé de lecture afin de souligner l'entrée du lecteur dans le texte, en particulier sa fonction intercalaire entre la perception immédiate et le point de vue ; clé de lecture qui, une fois énoncée, permet un processus d'élaboration aboutissant à un point de vue interprétatif. Nous verrons plus loin que les interprétations s'élaborent dans la puissance structurante du débat interprétatif.

\section{Activités langagières et parcours interprétatifs}

Le carnet de lecture, ayant fait l'objet d'une analyse de contenu, est découpé en trois périodes ; chaque période correspondant à une activité interprétative régie par les interactions langagières.

- La première période est toute entière consacrée à la réception du texte, l'écriture du roman, le monde et la manière de recevoir le texte font l'objet de nombreux échanges. C'est une écriture en écho qui apparaît, libérant la perception des lecteurs aux prises avec le besoin de dire, voire de redire ce qui les affecte. Ainsi, l'émotion, l'évocation du monde du roman irriguent les écrits.

- La seconde période est le lieu d'un conflit interprétatif qui oppose les lecteurs pris par le monde du texte aux lecteurs ironiques démantelant les stéréotypes véhiculés, les 
procédés qui invitent au pathos. Cette rupture de consensus permet un élargissement de l'interprétation.

- La troisième période est caractérisée par une hétérogénéité interprétative. Chaque lecteur, averti de la diversité des réceptions, tient un positionnement nouveau ou maintient son ancien positionnement.

\subsection{Première période : du langage de l'émotion au monde du texte}

Du 20 octobre au 13 novembre 2003, les lecteurs proposent deux grands thèmes de réflexion qui feront l'objet de nombreuses reformulations. Dès les premières pages du carnet, les clés de lecture «langage / écriture » et «monde de la vie dans la rue » accaparent les lecteurs. Mais ces clés ne sont pas abordées pour elles-mêmes, l'analyse est court-circuitée par les résonances émotives ainsi que par la réflexion sur le rapport à la lecture du texte. La posture de lecteur affecté par les résonances du roman devient une clé de lecture à part entière, notamment lorsque le style choque une lectrice qui déclare ne pas vouloir relire le texte très réaliste qui provoque un "sentiment presque oppressant", mais qui reconnaît les effets du texte sur le lecteur, "la description de la vie dans la rue est plus que réaliste, elle fait réfléchir le lecteur sur ses propres actes».

Les lecteurs se positionneront donc par rapport à l'écriture du texte, au monde du roman en faisant de constants va et vient avec leur attitude de lecteur troublé. Le langage de l'émotion domine sans voiler pour autant la construction d'une référence commune concernant le monde construit par le roman. Les écrits qui suivent marquent le tissage progressif de la référence

Le 23 octobre,

"Contrairement à la première réflexion, je n’ai pas tenu compte des mots prononcés. J'ai été complètement prise par l'ambiance du texte, ce froid. Ce froid qui installe le décor, qui annonce la mort ; Et pourtant ce froid que Titi et Rico portaient en eux, déborde de chaleur humaine... »

Le 4 Novembre,

« on ne peut s'empêcher de continuer la lecture de ce livre, il nous amène à nous poser de multiples questions existentielles...»

Le 6 novembre,

"Contrairement à Anne - Laure, j'ai du mal à lire ce livre d'un seul coup. Je trouve les premiers chapitres très difficiles, très émouvants après avoir lu un chapitre, je laisse quelques jours pour digérer, pour prendre du recul. Je suis frappée du réalisme du texte. Habitant Marseille, après mes premières lectures, je voyais dans chaque SDF, un Rico, un Titi, un Dédé. La mort de Titi m'a beaucoup choquée. "Mort malade, mort de faim, mort de froid »

Le 12 novembre,

" Je suis aussi frappée que les autres du réalisme, de la dureté du livre, de cette façon crue de dire les choses....J'ai été très surprise de l'entrée en scène du "je». On se rend compte d'un coup que quelqu'un raconte cette histoire...petit à petit on sent une certaine admiration du narrateur pour son personnage principal, son héros...on observe également de plus en plus un rapport à la littérature et à la poésie avec st John Perse, et plus on avance plus il y a de la poésie qui donne sens à cette vie, à côté du ton cru et du narrateur de cette façon... »

Les interactions langagières se tissent sur un fond de consensus si bien qu'un certain pathos se dégage des premières pages du carnet de lecture. Les positionnements énonciatifs oscillent entre assertions (possibilité de lire ou pas) et modalisations relatives à l'atmosphère du roman. La volonté de distanciation devant la contagion de l'émotion caractérise le positionnement de certains lecteurs qui dégageront d'autres clés de lecture. L'écriture du roman, la réflexion sur l'auteur, les personnages, le narrateur émergeront dans ce flux émotif. 
Dans le tableau qui suit, nous avons répertorié les clés de lecture énoncées dans le carnet. Chaque ligne du tableau correspond à une double page du carnet de lecture. Les flèches figurent les interactions entre lecteurs ainsi que l'objet des interactions.

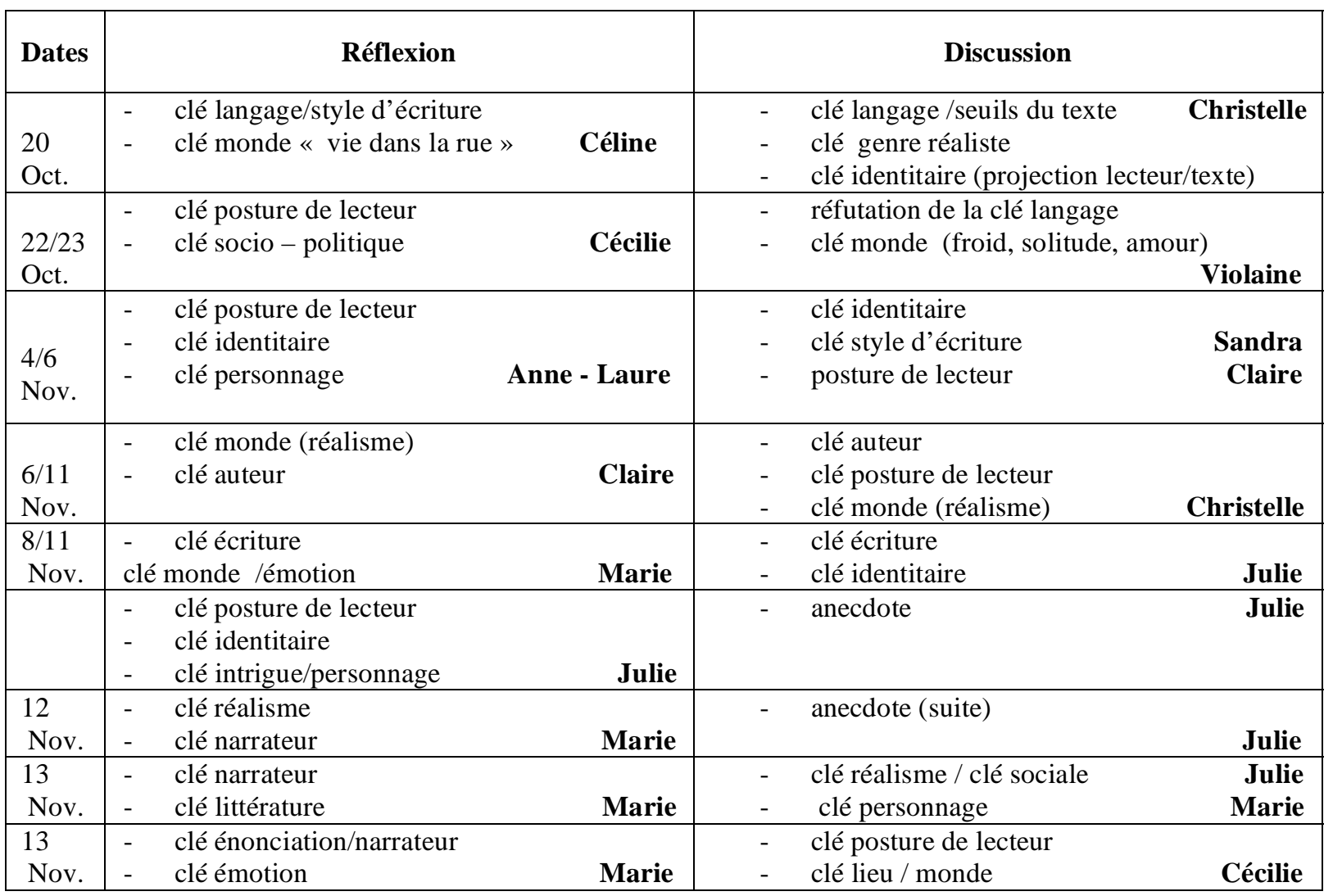

\section{2. - Seconde période : Style ironique et forme argumentative}

Cette seconde période (du 13 novembre au 26 novembre) est marquée par un conflit d'interprétation opposant les lectrices qui avaient déployé un langage empli d'émotion à Anthony qui maniera le discours argumentatif, reprenant les impressions pour mieux les retourner et en souligner la vacuité. La provocation est à son comble lorsque le lecteur critique recourt à l'ironie, cherchant à choquer, si ce n'est à remuer les lectrices gagnées par l'atmosphère pathétique du roman. Ainsi, sur un ton comique, le lecteur reprendra les clés de lecture qui avaient fait consensus pour en relever les failles. La syntaxe, la narration, les bons sentiments au rang desquels l'attachement pour la ville de Marseille seront systématiquement épinglés. L'écrit résonne comme un verdict tant il allie l'ironie à la forme argumentative.

\footnotetext{
«Dans ce livre, Izzo nous plonge et nous noie dans le sordide-et-triste-monde-de-la-rue-. A l'image de la dernière phrase du dernier chapitre "C'était la seule saloperie de putain de vérité de cette vie...». Tout le livre a une syntaxe aussi agréable à l'oreille qu'une symphonie de Schönberg, c'est dire si la lecture enchante l'ouie... Izzo raconte l'histoire de Rico qui sachant sa fin proche décide comme Aznavour d'aller crever au soleil. Quitte à mourir autant le faire en bronzant, logique. Mais le sud de la France est vaste, alors pourquoi Marseille? Izzo trouve l'astuce en racontant la première histoire d'amour de Rico avec Léa, Izzo se sert de Léa pour déclarer sa flamme à Marseille pendant un chapitre entier !...»"

"Vous me donnez l'impression qu'il ne faut pas se moquer de ce livre aux personnages creux, à l'intrigue inexistante, aux ficelles d'écriture trop voyantes...que de bons sentiments, je vais donc parler de la forme et $d u$ fond...il y a un manichéisme inquiétant chez Izzo... les héros sont des saints, des victimes, rien n'est mauvais en eux, même lorsque Rico braque les gens, il est gentil. C'est les autres, les méchants qui leur pourrissent la vie. Mais ne vous inquiétez pas, ils iront au paradis...»
} 
Le ton polémique ainsi que les arguments (parfois convenus : la référence à Schönberg paraît tout aussi discutable !) susciteront des réactions, elles sont visibles dans le tableau de la page suivante : le nombre d'interactions (voir les flèches qui convergent vers Anthony) qui s'adresseront au lecteur critique montre l'importance du débat interprétatif. Ainsi, sur le mode - de la concession, Julie tentera de répondre en essayant de défendre le paramètre du lieu dans la compréhension du roman «c'est vrai que les marseillais sont particulièrement attachés et fiers de leur ville... ».

- de l'opposition, Sandra avancera ses arguments « contrairement à Anthony, je n'ai pas perçu la description de Marseille comme un patriotisme démesuré... »

- de la distance, Marie écrit une semaine plus tard «je voudrais revenir sur Marseille pour compléter les remarques d'Anthony et d'Elodie. Il me semble que Marseille est vraiment le lieu du repos du guerrier... cette histoire est comme une grande épopée que Rico doit traverser avec des épreuves pour arriver à sa fin, au lieu du repos suprême : la ville de Marseille qui va en quelque sorte le protéger»

- de la distance toujours, Aurélie « je voudrais revenir sur les remarques d'Anthony et de Pierre, si j'ai bien compris ce que tu dis, tu vois dans le thème principal, la vie dans la rue, un prétexte pour l'auteur pour parler de Marseille. Peut - être... mais pour ma part, je ne pense pas. J'ai l'impression et ça me chagrine pour toi que tu es passé à côté de l'image dominante du texte : la vie des marginaux et leur poésie »., " Pour Pierre... J'ai l'impression qu'il y a deux types de lecteur dans ce carnet de lecture : ceux qui ont accepté de regarder et de laisser ouverte la fenêtre qu' Izzo nous ouvre sur des gens tout proches de nous et que nous pourrions être et ceux qui ont jeté un coup d'œil. »

\begin{tabular}{|c|c|c|c|}
\hline Dates & Réflexion & & Discussion \\
\hline $\begin{array}{l}14 / 18 \\
\text { Nov. }\end{array}$ & - clé monde / affect & Cécilie & $\begin{array}{l}\text { - clé monde « graal du trottoir » } \\
\text { - clé émotion } \\
\text { - clé genre discursif }\end{array}$ \\
\hline $\begin{array}{l}18 \\
\text { Nov. }\end{array}$ & $\begin{array}{l}\text { - clé émotion } \\
\text { - fils conducteurs : alcool, froid } \\
\text { - clé émotion }\end{array}$ & Claire & $\begin{array}{lr}\text { - clé monde/fatalité } & \text { Anne-Laure } \\
\text { - clé émotion } & \text { Julie }\end{array}$ \\
\hline $\begin{array}{l}19 \\
\text { Nov. }\end{array}$ & $\begin{array}{l}\text { - clé critique du monde } \\
\text { - clé critique de l'écriture } \\
\text { - clé critique de l'écrivain }\end{array}$ & Anthony & $\begin{array}{l}\text { - clé monde } \\
\text { - clé identitaire }\end{array}$ \\
\hline $19: 11$ & $\begin{array}{l}\text { - clé posture de lecteur } \\
\text { - clé énonciation - narrateur } \\
\text { - clé monde / alcool }\end{array}$ & Sandra & $\begin{array}{l}\text { - clé monde } \\
\text { - clé auteur } \\
\text { - clé écriture }\end{array}$ \\
\hline $\begin{array}{l}19 / 11 \\
25 / 11\end{array}$ & $\begin{array}{l}\text { - clé monde /décrochage social } \\
\text { - décrochage social } \\
\text { - clé émotion } \\
\text { - clé émotion } \\
\text { - clé monde } \\
\text { - clé monde } \\
\text { - clé socio -politique }\end{array}$ & $\begin{array}{r}\text { Pierre } \\
\text { Claire } \\
\text { Violaine } \\
\text { Elodie }\end{array}$ & 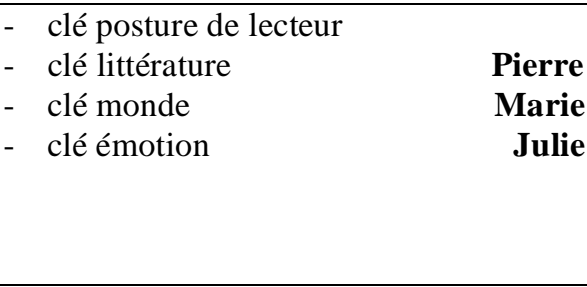 \\
\hline $\begin{array}{l}25 \\
\text { Nov. }\end{array}$ & $\begin{array}{l}\text { - clé posture de lecteur } \\
\text { - clé structure du texte } \\
\text { - clé langage }\end{array}$ & Marie & $\begin{array}{ll}- & \text { clé posture de lecteur } \\
\text { - clé monde «vie des marginaux et de } \\
\text { leur poésie » }\end{array}$ \\
\hline $\begin{array}{l}26 \\
\text { Nov. }\end{array}$ & - clé posture de lecteur & Aurélie & $\begin{array}{lr}\text { - clé identitaire } & \text { Aurélie } \\
\text { - clé posture de lecteur } & \text { Julie }\end{array}$ \\
\hline
\end{tabular}




\subsection{Troisième période : Les horizons interprétatifs}

$\mathrm{Au}$ cours de cette troisième période (du 26 novembre au 17 décembre), après le moment de conflit interprétatif, les interactions langagières sont traversées par une approche compréhensive : la part de l'écriture est soulignée pour saisir l'importance de la clé monde de la rue, «c'est vrai qu'en passant par une description détaillée des villes, on est frappée par la réalité du texte, des personnages ». Le genre descriptif est évoqué pour mieux revenir au monde du roman, les interactions langagières sont centrées sur cet aspect : pour une lectrice, le monde de la rue suppose d'aborder le problème sous l'angle social, pour une autre, sous l'angle de références sociologiques avec Bourdieu, pour une autre encore sous l'angle politique. Les déplacements de significations renvoient différents positionnements qui conduiront deux lectrices à interroger l'importance du titre du roman. Un nouveau conflit intervient à l'initiative du même lecteur critique, lequel s'insurge contre la mièvrerie sanglotante du récit. Un résumé du livre sous une forme décapante soulève une nouvelle fois de l'opposition, puis chaque lectrice revient à son positionnement insistant sur la dureté de l'histoire et sur l'émotion qui a surgi à la lecture du roman.

Dans cette dernière phase, les lecteurs maintiennent leur positionnement, chaque lecteur avance son idée sous forme assertive. Il n'est plus temps d'interagir et le débat ayant eu lieu, des horizons interprétatifs coexistent.

\begin{tabular}{|c|c|c|c|c|}
\hline & Réflexion & & Discussion & \\
\hline 19 & - clé monde & & - clé monde-clé écriture & \\
\hline $26 \mathrm{~N}$ & - clé écriture & Cécilie & - clé personnage & Julie \\
\hline 20 & - clé sociale & Céline & - clé monde & Julie \\
\hline 21 & - clé sociologique & Julie & - clé monde & Julie \\
\hline 22 & - clé émotion & Céline & $\begin{array}{l}\text { - clé référence poétique } \\
\text { - intertextualité } \\
\text { - clé monde }\end{array}$ & Anthony \\
\hline 23 & - clé émotion (suite) & Céline & $\begin{array}{l}\text { - clé ironie émotive } \\
\text { - clé critique écriture }\end{array}$ & Anthony \\
\hline $\begin{array}{c}24 \\
4 \\
\text { Déc. }\end{array}$ & $\begin{array}{l}\text { - clé politique } \\
\text { - clé émotion }\end{array}$ & Céline & $\begin{array}{l}\text { - clé émotion } \\
\text { - clé politique } \\
\text { - clé émotion }\end{array}$ & $\begin{array}{l}\text { Cécilie } \\
\text { Claire }\end{array}$ \\
\hline 25 & - clé personnage & Céline & $\begin{array}{l}\text { - clé émotion } \\
\text { - clé seuils du texte } \\
\text { - clé seuils du texte }\end{array}$ & $\begin{array}{r}\text { Sandra } \\
\text { Adeline }\end{array}$ \\
\hline $\begin{array}{l}26 \\
9 \\
\text { Déc. }\end{array}$ & - clé monde & Violaine & $\begin{array}{l}\text { - clé posture de lecteur } \\
\text { - clé posture de lecteur }\end{array}$ & $\begin{array}{l}\text { Aurélie } \\
\text { Elodie }\end{array}$ \\
\hline 27 & $\begin{array}{l}\text { - clé narration } \\
\text { - clé critique narrative }\end{array}$ & $\begin{array}{r}\text { Claire } \\
\text { Anthony }\end{array}$ & - clé critique narrative & Anthony \\
\hline 28 & - clé critique narrative (suite) & Anthony & $\begin{array}{l}\text { - clé critique narrative } \\
\text { - clé personnage }\end{array}$ & $\begin{array}{l}\text { Adeline } \\
\text { Sandra }\end{array}$ \\
\hline 29 & - clé personnages & Anthony & & \\
\hline 30 & - clé personnage (suite) & Anthony & & \\
\hline 31 & - clé posture de lecteur & Adeline & $\begin{array}{l}\text { - clé personnage } \\
\text { - clé politique }\end{array}$ & Adeline \\
\hline
\end{tabular}




\section{Vers un mouvement interprétatif collectif}

La réception du roman appelle des positionnements particuliers, une mosaïque de perceptions semble courir sur les premières pages du carnet. Cependant, loin de figurer comme éléments disparates, les réflexions des lecteurs s'élaborent dans un mouvement plus global et il devient possible d'analyser le cheminement du processus interprétatif. Le tissage de l'interprétation dépend de quatre processus langagiers : la désignation de clés de lecture, la reformulation de certaines clés, le déplacement des significations, l'amplification thématique.

\section{1 - La désignation des clés de lecture}

Dès la première page du carnet, deux clés de lecture sont proposées par une lectrice qui fait part de ses impressions : elle souligne ainsi l'importance du langage, clé particulièrement importante dans le roman et se dit «frappée par la description faite de la vie dans la rue » apportant cette fois l'idée d'un monde spécifique. En inaugurant de la sorte le carnet de lecture, la lectrice suscitera d'autres réflexions qui prolongeront les premières impressions. Ainsi, au cours de l'interaction suivante, une lectrice reprend les deux clés de lecture précédentes, se déclare impliquée par cette histoire, affichant sa projection dans l'univers du roman pour s'adresser ensuite des questions inédites. Ce positionnement de lecteur s'apparente à un processus intro - projectif, dans lequel un questionnement existentiel est mobilisé. Le plan identitaire et la perspective socio -politique s'entremêlent si bien que le chercheur est dans un dilemme pour identifier la clé de lecture qui semble tenir de la posture de lecteur. A ces premières clés, les lecteurs grefferont la clé émotion, clé qui découle de la réception du roman et que les lecteurs affichent dans un second temps, une fois que les clés langage -écriture, monde, posture de lecteur ont été avancées. De ce fait, l'émotion tient une place importante dans les interactions entre lecteurs. Le lecteur se dévoile peu à peu et ose manifester puis partager ses émotions.

Les réflexions qui mobilisent les lecteurs visent à qualifier l'univers du roman, l'ambiance du livre. Dès les premières pages du carnet de lecture, les interactions portent sur le froid, la mort, l'alcool, la misère, la ville de Marseille. A plusieurs reprises, les lecteurs s'interpellent pour confirmer un point de vue ou le nuancer, partager une émotion. La désignation des clés de lecture lance le processus interprétatif qui sera ensuite prolongé par la recherche de l'interprétation juste à travers l'atteinte de l'idée précise, du mot juste.

\section{2 - Jeux de reformulations langagières}

Le mouvement interprétatif prend de l'ampleur avec les reprises langagières qui sont tissées autour des clés de lecture. Le processus interprétatif est alimenté par les jeux de reformulations avec les termes décor - ambiance - lieu - réalisme - vie dans la rue - univers inconnu de la rue - monde des SDF qui renvoient à la clé monde.

\footnotetext{
«En complément des propos de Claire et de Violaine, je voulais souligner que ce livre m'a plongé littéralement dans l'univers inconnu de la rue. »

- «, le décor choisi n'est pas fortuit mais représenté dans toute sa beauté car c'est la dernière lueur d'espoir de Rico. »

- « je voudrais revenir sur l'ambiance qui règne dans le livre. J'ai été frappée par cette violence omni présente qui prend une place de plus en plus forte. »

- « je ne suis pas d'accord! La vie de cet homme est triste, malheureuse et ennuyeuse, on ne peut pas lui inventer une vie extraordinaire ! La réalité ! C'est ce que veut traduire Izzo »
} 
De même, la clé langage - écriture prend de l'épaisseur avec l'apparition d'autres clés comme celles du narrateur, de la psychologie des personnages, de la littérature, de la structure du texte, des seuils du texte.

- «Comme Claire, j'ai voulu savoir qui était cet auteur

- Petit à petit, on sent une certaine admiration du narrateur à l'égard de son personnage principal, de son héros. Il y a une progression qui fait que plus on avance, plus on sent le narrateur présent... » Marie

- "Vous me donnez l'impression qu'il ne faut pas se moquer de ce livre aux personnages creux, à l'intrigue inexistante et aux ficelles d'écriture trop voyantes pour la sacro-sainte raison qu'il traite des malheureux et de la misère. Que de bons sentiments » Anthony

- Izzo, lui-même a dû faire preuve d'empathie pour écrie ce livre. Comme le dit Pierre, on dirait une étude sociologique. Le sociologue doit lui aussi faire preuve d'empathie comme le dit Bourdieu dans un texte qui s'appelle «Comprendre » extrait du recueil d'entretiens «La misère du monde » (1998). Cela traite de cette misère sociale, de cette souffrance sous la forme de récits de vie / entretiens et de leur interprétation.. »Julie

Quatre lecteurs citent de courts passages du roman soulignant ainsi une syntaxe particulière «mort de maladie, mort de faim, mort de froid » ou une atmosphère qui renvoie au monde du roman. «Mets-toi bien ça dans la tête, Rico, tendre la main c'est admettre qu'on est hors circuit, qu' on ne s'en sortira plus. »

\section{3 - Déplacements de significations}

Les déplacements de significations sont particulièrement observables en ce qui concerne la réflexion sur l'écriture et sur le monde du roman. Ces déplacements se réalisent sous deux modalités, soit par consensus, soit par contradiction. Les lecteurs mettent à l'épreuve ces clés en cherchant des significations contiguës. En cela, les déplacements constituent des modes d'appropriation du roman. En guise d'exemple, nous proposerons la clé monde de la vie dans la rue :

\section{Le 20 octobre}

- je suis frappée par la description faite de la vie dans la rue. J'en retiens une exacerbation des sentiments liés à la vie et à la mort. Il y a d'un côté une extraordinaire liberté à être SDF et en même temps, il y a, encore plus réelle, l'idée de la mort, de la fin inéluctable car ce choix de vie peut consister en une forme de lent suicide. (Céline)

Le $23 / 10$

- «j'ai été complètement prise par l'ambiance du texte, ce froid. Ce froid qui installe le décor, qui annonce la mort. Et pourtant, ce froid que Titi portait en lui et Rico ensuite, déborde de chaleur humaine que ces deux amis diffusent. Ce texte bascule dans le froid, la solitude de Rico, ...»(Violaine)

Le $9 / 11$

- j'ai pas tout compris, un peu comme si on se perdait dans les pensées de Rico ou dans celles du narrateur. Comme si la vie de Rico perdait de sa consistance, même dans sa tête. » Julie

le $14 / 11$

- Par rapport aux propos, réflexions et discussions précédentes, je replonge dans les premiers chapitres du livre, au moment magique de la découverte de Marseille par Rico...Marseille et son apéro, ses balades au Panier, ses habitants qui parlent fort, rient fort et tutoient beaucoup sûrement autant qu'ils klaxonnent!...j'aime Marseille dans le regard d'Izzo, vivante, tolérante, rebelle, pleine de Le 18/11 contradiction. (Cécilie)

- «Toutes ces bouteilles d'alcool me donnent la nausée » Pierre

- Enfin quelqu'un qui parle de ces bouteilles d'alcool «c'est par cela que je voulais commencer ce soir. Cette omniprésence de la boisson m'a un peu dérangée, mise mal à l'aise. je crois qu'elle me fait 
peur...j'ai l'impression que l'alcool et le froid sont les fils conducteur de ce livre.. j'arrive à la fin du livre et l'autre chose dont je voulais parler c'est l'espoir, j'ai eu du mal à trouver une lueur d'espoir dans la vie de Rico... (Claire)

- J'ai été également surprise par la quantité d'alcool et de cigarettes ingérées par les personnages. Mais en lisant ce livre, je comprends mieux leur utilité, ça aide à oublier les malheurs de la vie et à garder l'espoir nécessaire pour vivre (Sandra)

- «le décrochage social est souvent le fait d'une grande sensibilité retournée contre soi. Etre tout entier ramassé, retourné, comme un désarroi profond. L'alcool floue les limites individuelles et fond l'espace Le $30 / 11$ qui sépare de l'autre jusqu'à la fusion.

- "Izzo lui-même a dû faire preuve d'empathie pour écrire ce livre comme le dit Pierre, on dirait une étude sociologique, le sociologue doit lui aussi faire preuve d'empathie - compréhensive comme l'explique Bourdieu dans un texte qui s'appelle "Comprendre », extrait du recueil d'entretiens dans La misère du Monde. Cela traite de cette misère sociale, cette souffrance, sous la forme de récits des vielentretiens et de leur interprétation (pas que les SDF), tout un kaléidoscope social de ceux qui sont touchés par la crise, par la misère de position, misère de condition. » Julie

\section{4 - Amplification thématique}

Le mécanisme d'amplification découle de la recomposition thématique d'une clé de lecture, reformulée précédemment par les lecteurs et qui prend, à un moment donné du processus interprétatif, une valeur thématique plus globale. Tournant autour de la clé de lecture «monde de la vie dans la rue», les lecteurs interagissent et produisent des déplacements de significations. Ainsi, Pierre évoquera «la quête du Graal », Julie s'attachera à « la misère sociale », Claire à « la vie des SDF », Claire considérera que « l'alcool et le froid sont les fils conducteur de ce livre » jusqu'au moment où Pierre, sur la base des échanges antérieurs, proposera une nouvelle thématique. Ainsi, l'idée du « décrochage social » apparaît après de nombreuses interactions autour de l'atmosphère du roman. En synthétisant les approches précédentes Pierre énonce une clé qui les intègre et les dépasse. Le processus interprétatif est ici opérant par amplification des significations déposées dans le carnet de lecture :

- «le décrochage social est souvent le fait d'une grande sensibilité retournée contre soi. Etre tout entier ramassé, retourné, comme un désarroi profond. L'alcool floue les limites individuelles et fond l'espace qui sépare de l'autre jusqu'à la fusion. » Pierre

Plus loin dans le carnet de lecture, ce thème fera à nouveau l'objet d'un déplacement après qu'une lectrice ait tenu un plaidoyer pour une lecture plus nuancée du roman d'Izzo. L'amplification thématique est la suivante :

- « l'image dominante du texte : la vie des marginaux et leur poésie.... Etre dans la rue, c'est peut-être retourner à une liberté primaire, infantile, dénuée de tous codes et contraintes sociales. C'est peut-être là-dedans que résident leurs espoirs. » Aurélie

Le rapport proliférant aux significations, les tensions entre clés de lecture sont saisies en un thème. Un mois se sera écoulé avant que la thématisation de Pierre surgisse faisant résonner toutes les variations signifiées par les lecteurs. Ce processus d'amplification semble opérer après un certain nombre d'interactions langagières et dans un espace - temps partagé. 


\section{EPROUVER LE LITTERAIRE}

L'expérience interprétative dans laquelle les étudiants sont entraînés constitue une modalité de formation à la lecture littéraire. Les dix-huit lecteurs sont confrontés à des points de vue parfois proches, parfois hétérogènes. Cette pluralité des significations favorise une approche plus complexe du processus d'interprétation et permet de façon tangible de repérer les impasses d'une approche essentialiste dans laquelle les lecteurs versent parfois. Les traces langagières apparaissent sous des formes consensuelles, divergentes, polémiques. Le fait de découvrir la perception d'autres lecteurs permet le développement d'une réflexion sur les postures de lecteur. Ainsi, lors du débat qui a suivi l'écriture partagée, les formés soulignent la diversité des approches. C'est ainsi, que le groupe de lecteurs est en mesure d'identifier différents rapports à la lecture. Une première grande opposition surgit, une grande majorité d'étudiants a été entraînée par le pathos du roman tandis que deux lecteurs ont cherché à s'en dégager, y compris en faisant fronde et en opposant une lecture politique. Le groupe de formation prend conscience des différences de champs perceptifs mobilisés par les lecteurs. Cette première analyse est suivie d'une prise de conscience individuelle des postures de lecteur et devant la diversité de ces postures, le groupe revient sur l'idée d'une irréductible hétérogénéité interprétative.

\subsection{Des postures de lecteur}

L'analyse de l'expérience et la description des positionnements permettent le dégagement de quatre manières de lire le roman :

- La lecture adhésion : lecture de reconnaissance de son identité personnelle, de l'identité régionale dans laquelle on cherche le plaisir de la confirmation « je connais l'auteur, et puis il y a Marseille ». Le parti pris du lecteur est affiché, la subjectivité est une entrée majeure dans la réception du roman.

-La lecture compassionnelle : proche de la lecture précédente, cette manière de lire marque cependant une certaine distance dans le fait d'identifier les ficelles d'écriture qui ont fait l'objet de certaines interactions dans le carnet de lecture. Le lecteur marque une complaisance à l'égard du roman « je reconnais les faiblesses mais ces stéréotypes me conviennent, je m'y retrouve ».

-La lecture focalisée : la lecture focalisée concerne plusieurs lectrices qui recherchent dans toute lecture la confirmation d'une attente précise. Pour certaines le langage devient une clé de lecture, pour d'autres la part de l'émotion est essentielle. Le positionnement du lecteur est particulièrement finalisé.

-La lecture interrogative : cette lecture est possible lorsque le lecteur reprend des paramètres du roman, pour en interroger la puissance évocatrice. Le réalisme si souvent évoqué est source de réflexions. Ce travail réflexif peut être tourné vers le lecteur dans des moments d'interrogation existentielle ou être élargi au champ social, voire politique.

L'identification des manières de lire renvoie aux lecteurs les postures distanciées ou transférentielles à partir desquelles ils ont agi. Ce temps d'évaluation occasionne une interrogation sur la posture de lecteur interprète d'un texte. La lecture littéraire accueillerait ces moments de réception et de construction d'une interprétation distanciée du texte. Il s'agirait alors de circuler entre plusieurs postures en évitant justement la lecture adhésion, la lecture compassionnelle, la lecture focalisée ! 


\subsection{L'hétérogénéité interprétative en formation d'enseignants}

La lecture littéraire à travers l'écriture du carnet nous permet d'envisager plusieurs pistes pour la formation. L'expérience vécue par les lecteurs met en évidence l'importance des positionnements adoptés pendant la lecture du roman. La lecture compassionnelle, la lecture adhésion, la lecture focalisée constituent des obstacles particulièrement importants si la visée est de former à la réception dans ses différentes modalités, d'éprouver la lecture littéraire comme un jeu de tensions ou de prolifération de significations. Les lecteurs ont pris conscience de l'ancrage qui était le leur et de la nécessité de circuler entre plusieurs postures. Cette expérience interroge la place accordée à la connaissance des théories et la mise en acte de ces apports dans l'action. Il s'agit ici de susciter la réflexion du praticien, de valoriser le rapport de congruence entre le su et l'agi, ce qui dans le champ de la professionnalisation conduit à interroger la posture du praticien réflexif (Schön, 1994).

Dans la présente recherche, les pratiques langagières constituent une entrée majeure dans le processus de formation. Les lecteurs ont interagi dans des formes discursives appropriées aux significations qu'ils déposaient dans le carnet. Lorsque l'atmosphère du roman était évoquée, l'écriture allusive et le questionnement traversaient les traces écrites, lorsque le débat interprétatif battait son plein, les arguments étaient avancés pour réfuter une assertion, enfin lorsqu'il s'agissait d'affirmer un positionnement, une certaine distance était gagnée à travers les reprises anaphoriques. La réflexivité du langage travaillait sur deux plans : le lecteur exposant son point de vue veillait à la forme discursive qu'il consignait dans le carnet.

Si l'écriture, par les interactions langagières et l'effet réflexif qu'elle produit, constitue à notre sens une instance de formation féconde, une autre voie de formation apporterait des effets prometteurs. La vidéo - formation, à partir de séquences de lecture littéraire vécues par les stagiaires, offrirait des possibilités de formation, à condition toutefois que le rapport à l'image n'entraîne pas d'effets identitaires déstabilisants chez les formés. Dans cette proposition, l'objectif est le même, il s'agit de former à la lecture littéraire dans un rapport de congruence entre le savoir théorique et la dimension praxéologique.

La recherche qui fait l'objet de cet article, nous entraîne à penser que la formation à lecture littéraire gagnerait à être articulée à la formation expérientielle. 


\section{Bibliographie}

BAKHTINE, M. (1984) Esthétique de la création verbale. Paris, Gallimard.

BARICCO, A. (1996) Soie. Paris, Gallimard.

BARTHES, R. (1984) Le bruissement de la langue. Paris, Seuil.

BARTHES, R. ( 1973) Le plaisir du texte. Paris, Seuil.

BAUTIER, E. (1998) «Des genres du discours aux pratiques langagières ou des difficultés à penser le sujet social et ses pratiques » in GROSSMAN, F. (ed) Pratiques langagières et didactique de l'écrit. Hommage à Michel Dabène. p.145-156. Grenoble, Ivel - Lidilem.

BOURASSA, B., SERRE, F., ROSS, D. (1999) Apprendre de son expérience. Québec, Presses de l'université du Québec.

BUCHETON, D. (2002) «Lire, comprendre, interpréter, sans expliquer». Trema, n¹9, Montpellier.

DE CERTEAU, M. (1990) L'invention du quotidien. Arts de faire. 1. Paris, Seuil.

COURTOIS, B. et PINEAU, G. (1991) La formation expérientielle des adultes. Paris, La Documentation Française.

DUFAYS, J-L. ( 2002) «Les lectures littéraires : évolution et enjeux d'un concept ». Trema, $\mathrm{n}^{\circ} 19$, Montpellier.

FRANCOIS, F. (1998) Le discours et ses entours. Paris, L'Harmattan.

GENETTE, G. (1987) Seuils. Paris, Seuil.

ISER, W. (1976) L'acte de lecture. Théorie de l'effet esthétique. Bruxelles, Mardaga.

JAUSS, H.R. (1978) Pour une esthétique de la réception. Paris, Gallimard.

JORRO, A. ( 2001) Les coulisses de la lecture interprétative. La tâche et ses entours. Actes du colloque DFLM, Neuchâtel, sur Cdrom.

JORRO, A. ( 1999) Le lecteur interprète. Paris, PUF.

JOUVE, V. (2001) Poétique des valeurs. Paris, PUF

RICOEUR, P. (1986) Du texte à l'action. Paris, Seuil.

SCHÖN, D. (1994) Le praticien réflexif. Montréal, Logiques.

TAUVERON, C. (2002) Lire la littérature à l'école. Paris, Hatier.

TERWAGNE, S., VANHUlLE, S., LAFONTAINE, A. (2003) Les cercles de lecture. Bruxelles : De Boeck-Wesmael.

VYGOTSKY, L.V. (1985) Pensée et langage. Paris : Messidor. 